\title{
Role of Cancer Emboli as a Metastatic Core on the Growth of Brain Metastasis in Patients with Non-Small Cell Lung Cancer
}

\author{
Jinuk Kim ${ }^{1}$, Gyuseo Jung ${ }^{1}$, Hoon Gi Kim ${ }^{1}$, Jeong Yeon Kim², Geun Young Yang ${ }^{2}$, Young Zoon Kim ${ }^{1}$ \\ ${ }^{1}$ Division of Neuro Oncology and Department of Neurosurgery, Samsung Changwon Hospital, Sungkyunkwan University School of Medicine, Changwon, Korea \\ ${ }^{2}$ Department of Anesthesiology and Pain Medicine, Samsung Changwon Hospital, Sungkyunkwan University School of Medicine, Changwon, Korea
}

Received: January 18, 2020

Accepted: February 3, 2020

\section{Corresponding Author:}

Young Zoon Kim, M.D., Ph.D.

Division of Neuro Oncology and

Department of Neurosurgery,

Samsung Changwon Hospital,

Sungkyunkwan University School

of Medicine, 158 Paryong-ro,

Masanhoewon-gu, Changwon

51353, Korea

Tel: $+82-55-233-5241$

Fax: $+82-55-233-8040$

E-mail: yzkim@skku.edu

\section{Objective}

Brain metastasis (BM) was a common complication of patient with non-small cell lung cancer (NSCLC) and associated with a poor prognosis. The study was to evaluate the effect of cerebral infarction (CI) which was originated from cancer emboli on the risk of BM in NSCLC for preventive therapy strategy.

\section{Methods}

Three hundred seven patients with newly diagnosed NSCLC in our institute from July 2013 to July 2018 were retrospectively analyzed. The diagnostic criteria of CI refereed to Updated Criteria for Population-Based Stroke and Transient Ischemic Attack Incidence Studies for the 21st Century. Depending on magnetic resonance imaging (MRI), the patients were divided into the BM group and control group (without $\mathrm{BM}$ ). Then, the prevalence of $\mathrm{CI}$ and baseline clinicopathological parameters were evaluated and compared between the two groups.

\section{Results}

Of the 307 patients, 204 patients (66.4\%) had CI and 52 patients (16.9\%) had BM. Especially, the prevalence of CI in the NSCLC patients with BM was $84.6 \%$, which was significantly higher than that of $62.7 \%$ in the NSCLC patients without BM $(\mathrm{p}=0.002)$. Following univariate logistic regression analysis and the multivariate model, the results demonstrated that $\mathrm{CI}$ was a significant independent risk factor for BM in NSCLC (odds rate, 3.303; 95\% confidence interval, 1.437-7.593; $\mathrm{p}=0.005$ ). What's more, CI contributed to a worse prognosis in NSCLC patients with BM. Dynamical trace confirmed CI could promote BM in NSCLC patient.

\section{Conclusions}

CI could be associated with a metastatic tropism to the brain and then with an increased risk of BM in NSCLC patient. Therefore, targeted intervention of the metastatic core of CI could offer promising approach for the prevention, prognostic evaluation, and therapy of BM in NSCLC patients for better clinical outcome.

Keywords: Cancer emboli; Metastatic core; Non-small cell lung cancer; Brain metastasis; Cerebral infarction 


\section{INTRODUCTION}

Lung cancer remains one of the most frequently diagnosed cancers as well as the leading cause of cancer-related mortality worldwide, in which non-small cell lung cancer (NSCLC) constitutes $85 \%$ cases $^{2,20)}$. Unfortunately, in nearly $20 \%$ of NSCLC patients, brain metastasis $(\mathrm{BM})$ is already present at diagnosis, with up to $50 \%$ of patients developing BM throughout the disease course ${ }^{12,28)}$. $\mathrm{BM}$ in NSCLC complicates the clinical picture and portends a poor prognosis with median survival of 3-7 months ${ }^{12,28)}$. However, risk factors and underlying mechanisms that the most common site of distant metastasis of lung cancer is the brain remain the mystery and have not been well addressed. Thus, to best improve the overall survival (OS) and quality of life for NSCLC patients, it is highly significant to illustrate the clinical risk factors of target BM in NSCLC for prevention strategies and specific therapies.

The tumor metastases are formed by a complex interaction between cancer cells and microenvironment, which is the "seed-soil" hypothesis ${ }^{26,29)}$. The "seed-soil" hypothesis sets forth the concept that a conducive microenvironment, or metastatic core, is necessary for disseminating cancer cells to engraft distant sites ${ }^{17,23}$. The common functions of metastatic core include anchorage, survival support, protection from external insults, licensing proliferation and outgrowth ${ }^{3)}$. Long-term since, much attention has focused on the molecular and genetic factors of cancer cells as "seed" endowed metastatic advantage. Meanwhile, the metastatic core creating a fertile "soil" for cancer cell to lodge and grow has been largely neglected. Therefore, the microenvironment of organs as the risk factors to determine metastatic colonization are particularly important for exploration, which might be most amenable to therapeutic interventions.

A very interesting one appears in several studies which demonstrated that patients with lung cancer were prone to induce cerebral infarction (CI) when compared with non-cancer control ${ }^{4)}$. What's more, $\mathrm{CI}$ occurrence could worse patient's prognosis in advanced or post-operative recurrent $\mathrm{NSCLC}^{14}$. These achievements inspire us from the perspective of "soil" to draw attention to the effect of $\mathrm{CI}$ as metastatic core on the risk of target-specific BM in NSCLC patients in the study, which may lead to detect therapeutic strategies and prevent metastasis at its earliest inception. The aim of this study is to evaluate the effect of $\mathrm{CI}$ which was originated from cancer emboli on the risk of BM in NSCLC for preventive therapy strategy.

\section{PATIENTS AND METHODS}

\section{Study population}

We conducted a retrospective study of all newly diagnosed NS-
CLC patients who were continuously admitted to Sungkyunkwan University Samsung Changwon Hospital from July 2013 to July 2018. The inclusion criteria were as follows: 1) all patients with histologically confirmed NSCLC, 2) did not receive any surgery, chemotherapy, radiotherapy, molecular targeted therapy, or immunotherapy in other hospitals before admission, 3) for the purpose of detecting distant organ metastasis, all patients accepted brain, chest, abdominal and pelvic imaging, such as ultrasound, computed tomography (CT), magnetic resonance imaging (MRI) and positron emission computed tomography/computed tomography (PET/CT), 4) complete medical records. The exclusion criteria were as follows: 1) NSCLC patients with synchronous distant metastases except for brain, 2) patients with NSCLC accompanied by malignant tumors in other parts of the body, 3) patients with CI caused by other pathogenic factors. In total, 307 patients were enrolled in this study.

\section{Data collection}

Data collected included age, gender, body mass index (BMI), histological type, and primary tumor size and location. In addition, laboratory tests were also covered, including D-Dimer (D-D) and the tumor markers of carcinoembryonic antigen (CEA) and carbohydrate antigen 125 (CA125). The survival time from the onset of BM were evaluated. The follow-up duration lasted until the death or July 31, 2019. OS from diagnosis of BM was evaluated.

The Institutional Review Board (IRB) of our hospital approved the study protocol (IRB number: SCMC 2019-02-015). All studies were conducted according to guidelines of the Declaration of Helsinki for biomedical research. Informed consent was waived due to its retrospective nature.

\section{The diagnosis of cerebral infarction}

The diagnostic criteria of CI refer to Updated Criteria for Population-Based Stroke and Transient Ischemic Attack Incidence Studies for the 21st Century8), which points out that $\mathrm{CI}$ can be diagnosed regardless of the duration of nervous symptoms/ signs when there are neuroimaging findings of responsible ischemic lesion. However, when no evidence of imaging evidence of responsible lesion can be obtained, the duration of symptoms and signs exceeding $24 \mathrm{~h}$ is still the time limit for diagnosis of ischemic stroke ${ }^{8)}$.

\section{Imaging evaluation of BM in NSCLC}

The patients had undergone a brain computed tomography (CT) scan. When brain lesion(s) could not be excluded, patients would undergo a magnetic resonance imaging (MRI). The diagnosis of BM on MRI was determined by two different neuroradiologists (YM Kim and MO Sunwoo) who were blinded to the clini- 
cal and pathological findings. Depending on the imaging examination results of CI and BM, NSCLC patients were divided into the $\mathrm{BM}$ group and control group (without $\mathrm{BM}$ ). Then, the prevalence of $\mathrm{CI}$ and baseline clinicopathological parameters were evaluated and compared between the two groups.

\section{Statistical analysis}

The prevalence of $\mathrm{CI}$ in the two groups was compared by the chi square test, other patient characteristics were compared either the chi square test, Fisher's exact test, or Wilcoxon two-sample test. Univariate and multivariate analyses were performed using logistic regression to assess the risk factors for BM. OS was plotted using the Kaplan Meier method. Differences in OS were analyzed using the log-rank test. $p<0.05$ was considered to be statistically significant. All statistical analyses were performed using SPSS version 25.0 software.

\section{RESULTS}

\section{Characteristics of NSCLC patient}

A total of 307 NSCLC patients ( 185 men and 122 women) with a median age of 64 years (range of 25-83 years) were confirmed for the analysis in our study. Among them, 52 patients (16.9\%) had $\mathrm{BM}$, and they were then divided into BM group. NSCLC patients without $\mathrm{BM}$ were regarded as the control group. In the 52 NSCLC patients with BM, 22 patients (42.3\%) underwent a brain CT or
MRI without any brain symptom, while other patients took the examination due to some symptoms, such as headache, hemiplegia and psychiatrical disorder. The summary of patients' characteristics of BM group and control group are shown in Table 1. The result demonstrated that patients with NSCLC are prone to induce CI with the prevalence of $66.45 \%$. Especially, the prevalence of CI in the NSCLC patients with BM was $84.6 \%$, which was significantly higher than that of $62.7 \%$ in the NSCLC patients without BM ( $p$ $=0.002, \chi 2$ test). In addition, there were significantly differences in primary tumor location (lobe), histology (type), D-D, CEA, and CA125 between BM group and control group. By contrast, there were no significant differences in age, BMI, sex, and primary tumor size between the two groups Table 1 .

\section{Risk factors for BM in NSCLC patient}

Following univariate logistic regression analysis, $\mathrm{CI}$, primary tumor location (lobe), histology (type), D-D, CEA, and CA125 were chosen as risk factors for BM in NSCLC. The results illustrated that BM was significantly associated with $\mathrm{CI}$ (odds rate [OR], 3.266; 95\% confidence interval [CoI], 1.475-7.231; $\mathrm{p}=0.004)$. Besides, adenocarcinoma $(\mathrm{p}=0.011)$, middle region tumor location $(p=0.005)$, increased CEA level $(p=0.002)$, increased CA125 level $(p<0.001)$ and higher D-D level $(p=0.027)$ were also linked with increased risks of BM in NSCLC Table 2.

Next, we assessed the significance of CI with respect to BM by using the multivariate model according to the factors that are prov-

Table 1. Baseline characteristics of NSCLC patients with/without BM

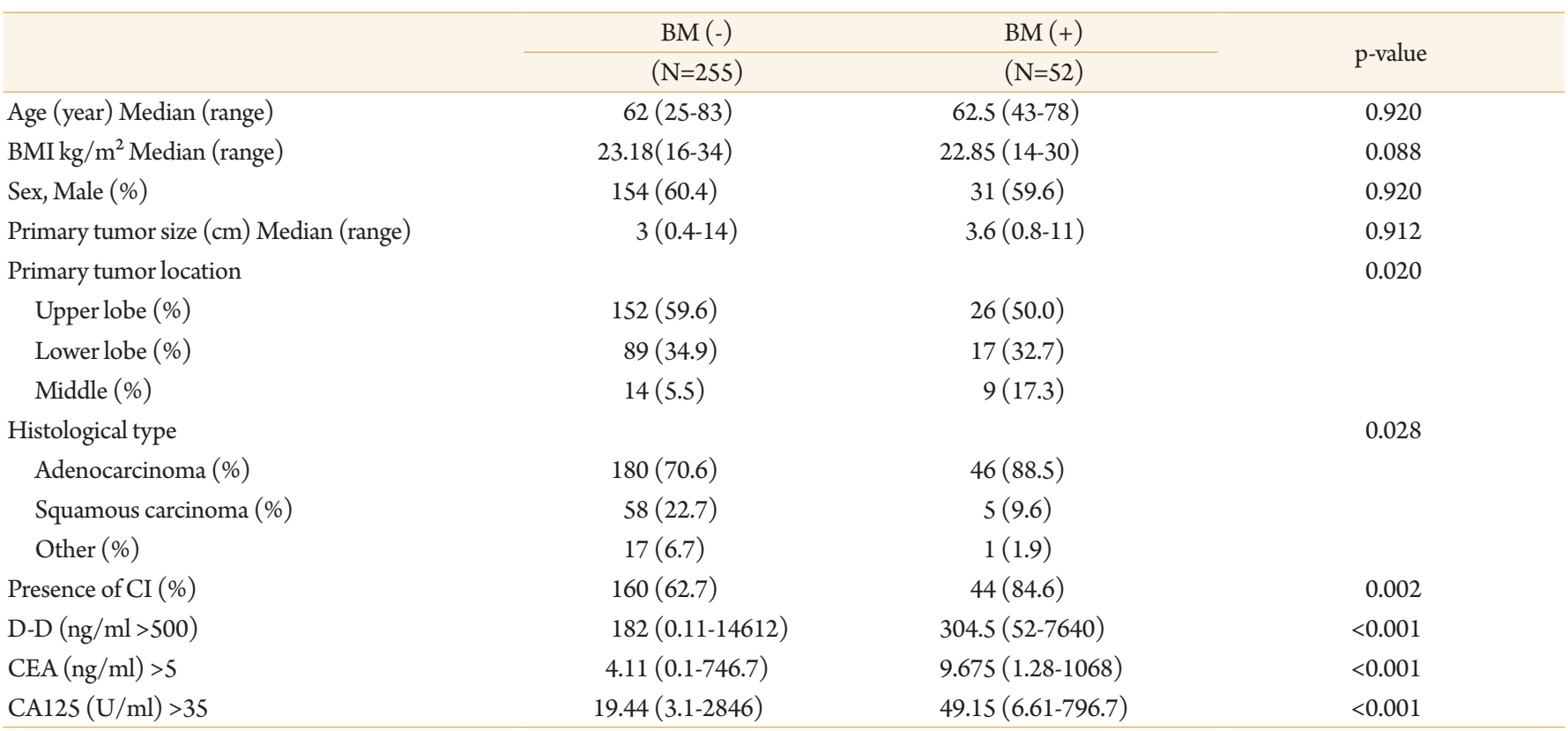

BM, brain metastasis; BMI, body mass index; CA125, carbohydrate antigen 125; CEA, carcinoembryonic antigen; CI, cerebral infarction; D-D, D-Dimer; NSCLC, non-small cell lung cancer. 
Table 2. Univariate analysis of risk factors associated with BM in NSCLC patient

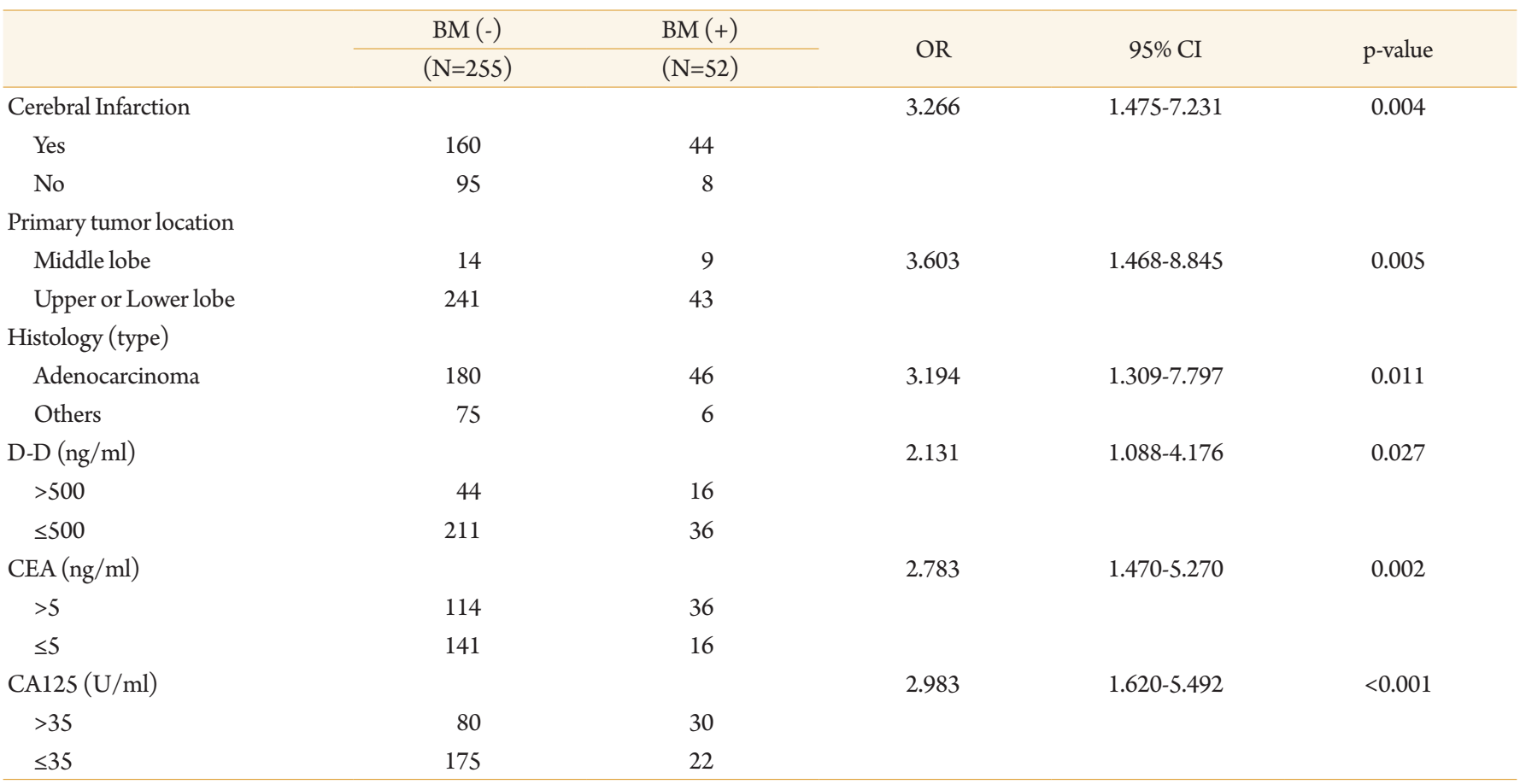

BM, brain metastasis; CA125, carbohydrate antigen 125; CEA, carcinoembryonic antigen; CI, confidence interval; D-D, D-Dimer; NSCLC, non-small cell lung cancer, OR, odd ratio.

Table 3. Multivariate analysis of risk factors associated with BM in NSCLC

\begin{tabular}{lccc}
\hline Variable & OR & $95 \%$ CI & p-value \\
\hline Cerebral Infarction (Yes vs No) & 3.303 & $1.437-7.593$ & 0.005 \\
Location (lobe) (Middle vs Not middle) & 3.105 & $1.153-8.364$ & 0.025 \\
Histology (Adenocarcinoma vs others) & 3.228 & $1.257-8.288$ & 0.015 \\
D-D $(\mathrm{ng} / \mathrm{ml})(>500$ vs $\leq 500)$ & 1.217 & $0.568-2.605$ & 0.613 \\
CEA $(\mathrm{ng} / \mathrm{ml})(>5$ vs $\leq 5)$ & 1.842 & $0.921-3.686$ & 0.084 \\
CA125 $(\mathrm{U} / \mathrm{ml})(>35$ vs $\leq 35)$ & 2.332 & $1.165-4.668$ & 0.017 \\
\hline
\end{tabular}

BM, brain metastasis; CA125, carbohydrate antigen 125; CEA, carcinoembryonic antigen; CI, confidence interval; D-D, D-Dimer; NSCLC, non-small cell lung cancer, OR, odd ratio.

en to be meaningful in the above univariate analysis. We could find that $\mathrm{CI}$ (OR, 3.303; 95\% CoI, 1.437-7.593; $\mathrm{p}=0.005)$ was a significant independent risk factor for BM in NSCLC Table 3. Besides, adenocarcinoma, middle region tumor location and increased CA125 level also remained as the risk factors for BM, whereas D-D, and tumor markers of CEA was no longer significant.

\section{Effect of cerebral infarction on survival of NSCLC patient with $\mathrm{BM}$}

To assess effect of CI on survival of NSCLC patients with BM, OS was measured and compared according to presence or absence of CI. The OS from diagnosis of BM was significantly shorter in NSCLC patients with CI than that in NSCLC patients with BM without CI ( $\mathrm{p}=0.024)$ Fig. 1 .

\section{Dynamically tracing of $\mathrm{CI}$ as metastatic core to promote BM of NSCLC}

A typical case with NSCLC who developed BM due to CI was illustrated in Fig. 2. In this case, when diagnosed with lung adenocarcinoma, one 79-year-old male patient underwent radical resection of the upper right lung cancer on November 24, 2015. Regular examination and follow-up were conducted in the outpatient department after the surgery. More than one year, a new patchy ischemic lesion was revealed at the right basal ganglia, which showed iso- or hypo-intense signal on T1weighted image (T1WI) of magnetic resonance (MR) on March 16, 2017 Fig. 2A. As time went 


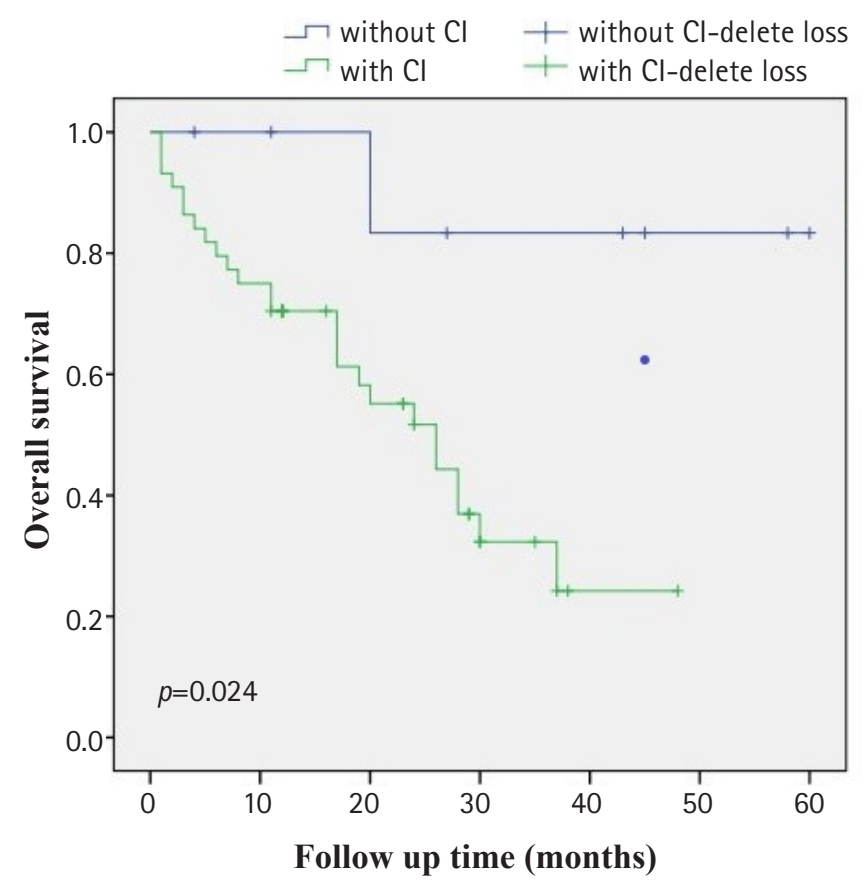

Fig. 1. Overall survival from diagnosis of BM in NSCLS patient. CI, cerebral infarction; BM, brain metastasis; NSCLC, non-small cell lung cancer. by, the lesion at the same site was significantly larger, which showed low signal on T1WI Fig. 2B when follow-up on November 10, 2017. Combined with the medical history, BM could not be excluded in the patient. Unfortunately, on March 16, 2018, the lesion obviously progressed, which showed an abnormal nodular enhancement lesion Fig. 2C. Thus, BM was confirmed in the NSCLC patient. The dynamic establishment of CI as metastatic core to promote BM of NSCLC was schemed in Fig. 2D. In brief, CI Fig. 2D creates a fertile "soil" for cancer cell as "seed" to lodge Fig. 2E and grow Fig. 2F.

\section{DISCUSSION}

Our study indicated that a higher prevalence of BM in NSCLC patients with $\mathrm{CI}$ compared to that in those without $\mathrm{CI}$, and $\mathrm{CI}$ was a dependent risk factor for BM in patients with NSCLC. Meanwhile, in NSCLC patients with BM, CI contributed to a worse prognosis in NSCLC patients with BM with shorter OS than that in those without $\mathrm{CI}$.

Cancer cells that disseminate from primary tumors are depen-
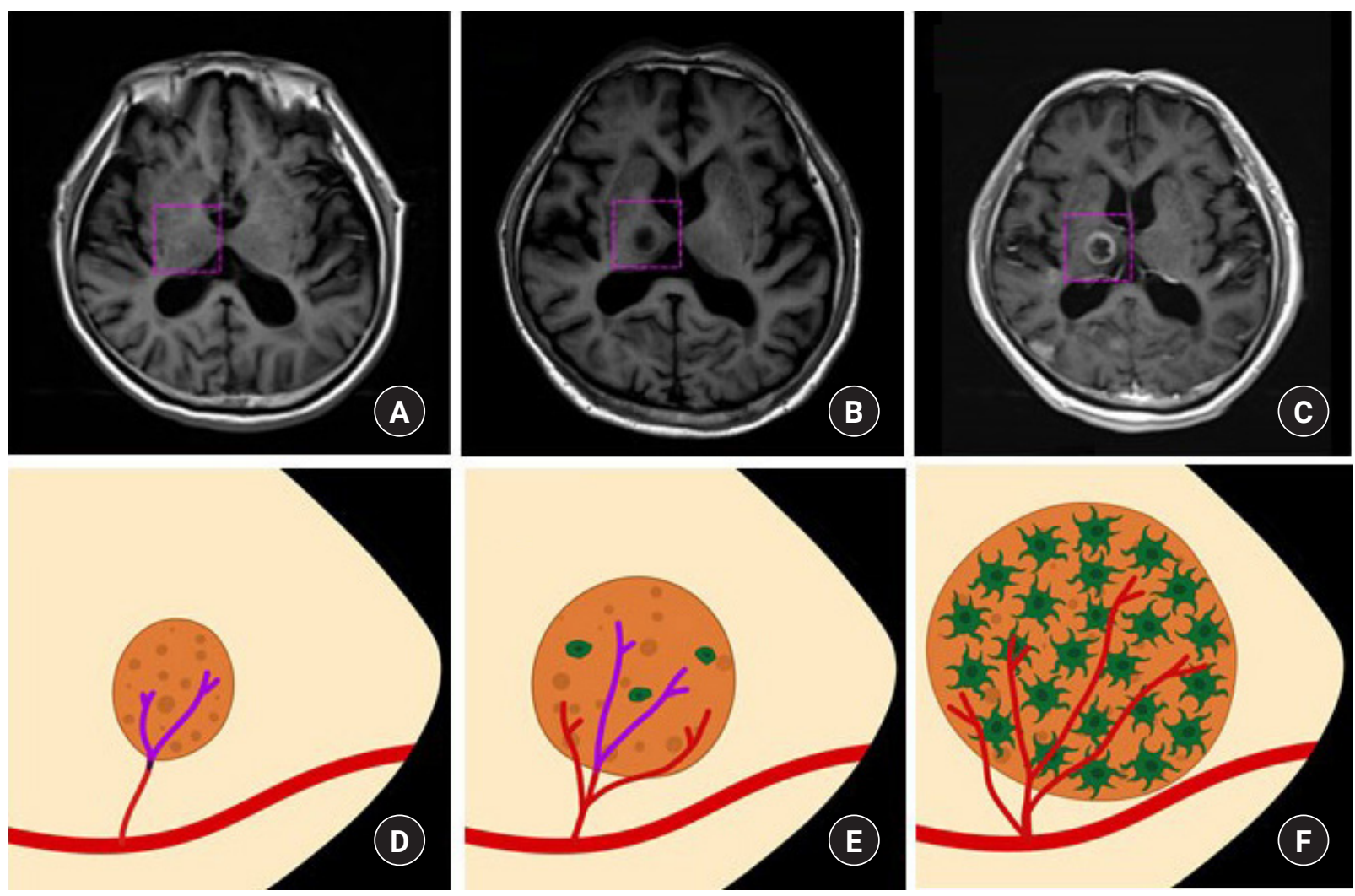

Fig. 2. Dynamically tracing of CI as metastatic cores to promote BM in NSCLC. A, MRI of CI; B, BM could not be excluded in MRI; C, confirmation of BM by MRI. B, the scheme of CI as metastatic core to promote BM in NSCLC. D, CI as a fertile "soil"; E, cancer cell as "seed" to lodge; F, metastatic colonization after proliferation and outgrowth.

CI, cerebral infarction; BM, brain metastasis; NSCLC, non-small cell lung cancer; MRI, magnetic resonance imaging. 
dent on the microenvironment they encounter at secondary sites which determine their fate. Metastatic core is the "soil" of the specific microenvironment established within the target organ to encourage the outgrowth of incoming the "seed" of cancer cells ${ }^{17,23)}$. It is very important to explore which microenvironment as metastatic core for BM in NSCLC due to its significant mortality.

It's a remarkable that the rate of cancer incidence is higher among ischemic stroke patients than the general population, ${ }^{9,13,21,22,25)}$, and cancer itself may increase a risk of CI occurrence ${ }^{10,11,16,30)}$. Once CI occurs in patients with cancer, neurological outcomes significantly worsen, and prognosis tends to be poor in addition to stroke-associated morbidity/mortality ${ }^{5,15,19)}$. Among all cancer types, patients with lung cancer have the highest incidence of $\mathrm{CI}^{4}$. Motoyasu et al. reported that development of $\mathrm{CI}$ contributed to a worse prognosis in patients with advanced and recurrent NSCLC ${ }^{20}$. In our study, we found that the incidence of synchronous BM was $16.9 \%$ in NSCLC, which was also in line with recent studies ${ }^{12,28}$. Our study also demonstrated a higher prevalence of $\mathrm{BM}$ in patients with $\mathrm{CI}$ at $84.6 \%$ compared to a prevalence of $62.7 \%$ in those without CI. All these findings shed us a light to explore a potential risk of $\mathrm{CI}$ to $\mathrm{BM}$ in patients with NSCLC.

To date, there have been few studies that focused on the relationship between $\mathrm{CI}$ and BM. A case report performed by Nielsen SL, et al. ${ }^{19)}$ showed that a patient with adenosquamous carcinoma of cervix developed BM limited to an area of evolving infarction. In another report, Sun YP, et al. ${ }^{27)}$ described a case that the patient complicated with $\mathrm{CI}$ and subsequent BM sarcoma after the initial cardiac tumor resection, which was confirmed by brain tissue pathology. In our study, we conducted a retrospective study of all newly diagnosed 307 NSCLC patients, and our results demonstrated that $\mathrm{CI}$ was a dependent risk factor for $\mathrm{BM}$ in patients with NSCLC.

$\mathrm{CI}$ accounts for $80 \%$ of cerebrovascular diseases ${ }^{8}$. The microenvironment of $\mathrm{CI}$ as metastatic core thus might be a crucial pillar to explain the specificity that applies to seeding and outgrowth of $\mathrm{BM}$ in NSCLC. CI causes the opening of the blood-brain barrier (BBB), active inflammation and edema formation ${ }^{1,6,18)}$. Under pathological condition of $\mathrm{CI}$, the $\mathrm{BBB}$ can be disrupted, followed by the extravasation of blood components into the brain parenchyma. So, metastasizing NSCLC cancer cells could be arrest in brain due to the BBB leakage. Furthermore, after initial cell death in CI, the clearance of debris in the lesion leaves a compartmentalized cavity that can accept a large volume transplant. Inflammation is an important driver of tumor development and metastasis ${ }^{24)}$. The inflammatory response also plays an important role in the pathological process of $\mathrm{CI}$, which is usually accompanied with the release of pro-inflammatory cytokines, such as tumor necrosis factor- $\alpha$
(TNF), interleukin 6 [IL-6], IL-1 $\beta$, monocyte chemotactic protein 1 , macrophage inflammatory protein $1 \alpha$ ), microglial activation, and adhesion molecules ${ }^{17)}$. The establishment of an inflammatory microenvironment in $\mathrm{CI}$ brain, either prior to or at the same time as the arrival of circulating tumor cells, is helpful in the seeding, survival, and proliferation of tumor cells in the metastatic core. Therefore, the roles of the microenvironment of $\mathrm{CI}$ are basically consistent with the common functions of different metastatic cores: anchorage, survival support, protection from external insults, licensing proliferation and outgrowth. In our study, dynamically tracing of $\mathrm{CI}$ as metastatic core to promote $\mathrm{BM}$ was confirmed in NSCLC patient.

Although our presenting study suggested interesting results of the metastatic core making cerebral infarction and its clinical roles, the major limitation of this study is the absence of histological analysis of the brain lesions. The diagnosis of $\mathrm{CI}$ was just performed in the radiological analysis with MRI without histological examination. In fact, we tried to reduce the bias on the diagnosis of CI by the individual 2 radiologists who did not have any information of the patients. In order to give a general practice in the clinic, the further comprehensive study such as randomized clinical trial is essential. Additionally, the precise figures of cancer emboli were not examined scientifically. For example, the circulating tumor cell can be the answer of the question for the cancer emboli as the cause of the cerebral infarction in the NSCLC patients. In the case of acute cerebral infarction, the thrombus which was obtained by the mechanical thrombectomy using endovascular intervention technique can be good method to provide the sample to analysis of the cancer emboli in the NSCLC patients.

Briefly, metastases account for the majority of cancer deaths, and $\mathrm{BM}$ are the most lethal complication of NSCLC. Host factors determining metastatic colonization to secondary organs are particularly important for exploration. In our study, the results of the effect of CI on survival in NSCLC patients with BM demonstrated that OS was significantly shorter than that in those without $\mathrm{CI}$, suggesting that $\mathrm{CI}$ may contribute to a worse prognosis. Thus, in clinical practice, targeting the metastatic cores of CI may evolve into a promising avenue for therapy of BM in NSCLC, such as certain agent preventing the cancer cells from forming the embolus in the systemic circulations.

\section{CONCLUSION}

Cerebral infarction could act as metastatic core to promote BM in patients with NSCLC. Therefore, targeted intervention of cerebral infarction could open up new strategies for the prevention, prognostic evaluation, and therapy of in NSCLC patients with BM. 


\section{NOTES}

\section{Conflict of interest}

The authors report no conflicts of interest in this work.

\section{Acknowledgements}

We thank the following individuals: Seok-Hyun Kim, M.D., (Department of Internal Medicine, Samsung Changwon Hospital) for the effort to manage the patients; Young Min Kim, M.D., and Mi-Ok Sunwoo, M.D., (Department of Radiology, Samsung Changwon Hospital) for their review of the neuroradiological images; Eun Hee Lee, M.D., and Mee-Seon Kim, M.D., (Department of Pathology, Samsung Changwon Hospital) for their pathological review; Young Wook Kim, M.D., (Department of Biostatistics, Samsung Changwon Hospital) for assistance with the statistical analysis; and Tae Gyu Kim, M.D., (Department of Radiation Oncology, Samsung Changwon Hospital) for administering the radiotherapy detailed in this work.

\section{REFERENCES}

1. Bhasin A, Srivastava MVP, Mohanty S, Vivekanandhan S, Sharma S, Kumaran S, et al. Paracrine Mechanisms of Intravenous Bone Marrow-Derived Mononuclear Stem Cells in Chronic Ischemic Stroke. Cerebrovasc Dis Extra 2016;6:107-119.

2. Bray F, Ferlay J, Soerjomataram I, Siegel RL, Torre LA, Jemal A. Global cancer statistics 2018: GLOBOCAN estimates of incidence and mortality worldwide for 36 cancers in 185 countries. CA Cancer J Clin 2018;68:394-424.

3. Celià-Terrassa T, Kang Y. Metastatic niche functions and therapeutic opportunities. Nat Cell Biol 2018;20:868-877.

4. Chen PC, Muo CH, Lee YT, Yu YH, Sung FC. Lung cancer and incidence of stroke: a population-based cohort study. Stroke 2011;42:3034-3039.

5. Chung JW, Cho YH, Ahn MJ, Lee MJ, Kim GM, Chung CS, et al. Association of cancer cell type and extracellular vesicles with coagulopathy in patients with lung cancer and stroke. Stroke 2018;49:1282-1285.

6. Eckert A, Huang L, Gonzalez R, Kim HS, Hamblin MH, Lee JP. Bystander Effect Fuels Human Induced Pluripotent Stem Cell-Derived Neural Stem Cells to Quickly Attenuate Early Stage Neurological Deficits After Stroke. Stem Cells Transl Med 2015;4:841-851.

7. Emre U, Gunes T, Pinar I, Kokturk F, Liman E, Yağiz O. Evaluation of ischemic stroke patients with systemic cancer. Ideggyogy Sz 2018;71:178-183.

8. Feigin V, Norrving B, Sudlow CLM, Sacco RL. Updated Crite- ria for Population-Based Stroke and Transient Ischemic Attack Incidence Studies for the 21st Century. Stroke 2018;49:22482255.

9. Gon Y, Okazaki S, Terasaki Y, Sasaki T, Yoshimine T, Sakaguchi $\mathrm{M}$, et al. Characteristics of cryptogenic stroke in cancer patients. Ann Clin Transl Neurol 2016;3:280-287.

10. Grazioli S, Paciaroni M, Agnelli G, Acciarresi M, Alberti A, D'Amore $C$, et al. Cancer-associated ischemic stroke: A retrospective multicentre cohort study. Thromb Res 2018;165:3337.

11. Guo YJ, Chang MH, Chen PL, Lee YS, Chang YC, Liao YC. Predictive value of plasma (D)-dimer levels for cancer-related stroke: a 3-year retrospective study. J Stroke Cerebrovasc Dis 2014;23:249-254.

12. Hanibuchi M, Kim SJ, Fidler IJ, Nishioka Y. The molecular biology of lung cancer brain metastasis: an overview of current comprehensions and future perspectives. J Med Invest 2014;61: 241-253.

13. Karlińska AG, Gromadzka G, Karliński MA, Członkowska A. The activity of malignancy may determine stroke pattern in cancer patients. J Stroke Cerebrovasc Dis 2015;24:778-783.

14. Kato M, Shukuya T, Mori K, Kanemaru R, Honma Y, Nanjo Y, et al. Cerebral infarction in advanced non-small cell lung cancer: a case control study. BMC cancer 2016;16:203.

15. Kneihsl M, Enzinger C, Wünsch G, Khalil M, Culea V, Urbanic-Purkart T, et al. Poor short-term outcome in patients with ischaemic stroke and active cancer. J Neurol 2016;263:150156.

16. Lee EJ, Nah HW, Kwon JY, Kang DW, Kwon SU, Kim JS. Ischemic stroke in patients with cancer: is it different from usual strokes? Int J Stroke 2014;9:406-412.

17. Liu Y, Cao X. Characteristics and Significance of the Pre-metastatic Niche. Cancer Cell 2016;30:668-681.

18. Mohan Rajwani K, Crocker M, Moynihan B. Decompressive craniectomy for the treatment of malignant middle cerebral artery infarction. Br J Neurosurg 2017;31:401-409.

19. Nielsen SL, Posner JB. Brain metastasis localized to an area of infarction. J Neurooncol 1983;1:191-195.

20. Patel N, Wu P, Zhang H. Comparison of gefitinib as first- and second-line therapy for advanced lung adenocarcinoma patients with positive exon 21 or 19 del epidermal growth factor receptor mutation. Cancer Manag Res 2017;9:243-248.

21. Qureshi AI, Malik AA, Saeed O, Adil MM, Rodriguez GJ, Suri MF. Incident cancer in a cohort of 3,247 cancer diagnosis free ischemic stroke patients. Cerebrovasc Dis 2015;39:262-268.

22. Quintas S, Rogado J, Gullón P, Pacheco-Barcia V, Dotor García-Soto J, Reig-Roselló G, et al. Predictors of unknown can- 
cer in patients with ischemic stroke. J Neurooncol 2018;137: $551-557$.

23. Ren G, Esposito M, Kang Y. Bone metastasis and the metastatic niche.J Mol Med (Berl) 2015;93:1203-1212.

24. Sirniö P, Väyrynen JP, Klintrup K, Mäkelä J, Karhu T, Herzig $\mathrm{KH}$, et al. Alterations in serum amino-acid profile in the progression of colorectal cancer: associations with systemic inflammation, tumour stage and patient survival. Br J Cancer 2019; 120:238-246.

25. Selvik HA, Thomassen L, Logallo N, Næess H. Prior cancer in patients with ischemic stroke: the Bergen NORSTROKE study. J Stroke Cerebrovasc Dis 2014;23:919-925.

26. Spano D, Zollo M. Tumor microenvironment: a main actor in the metastasis process. Clin Exp Metastasis 2012;29:381-395.

27. Sun YP, Wang X, Gao YS, Zhao S, Bai Y. Primary cardiac sarcoma complicated with cerebral infarction and brain metastasis: A case report and literature review. Cancer Biomark 2017;21: 247-250.

28. Taillibert S, Le Rhun É. Epidemiology of brain metastases. Cancer Radiother 2015;19:3-9.

29. Valastyan S, Weinberg RA. Tumor metastasis: molecular insights and evolving paradigms. Cell 2011;147:275-292.

30. Wang JY, Zhang GJ, Zhuo SX, Wang K, Hu XP, Zhang H, et al. D-dimer $>2.785 \mu \mathrm{g} / \mathrm{ml}$ and multiple infarcts $\geq 3$ vascular territories are two characteristics of identifying cancer-associated ischemic stroke patients. Neurol Res 2018;40:948-954. 